\title{
Highly Ordered Carbon-Based Nanospheres with High Stiffness
}

Sehmus Ozden ${ }^{\dagger^{*}}$, Chandra Sekhar Tiwary ${ }^{\dagger}$, JianyuYao ${ }^{\dagger,+}$, Gustavo Brunetto ${ }^{\dagger, \#}$, Sanjit

Bhowmick $^{\dagger \dagger}$, Syed Asif ${ }^{\dagger \dagger}$, Robert Vajtai ${ }^{\dagger}$, and Pulickel M. Ajayan ${ }^{\dagger *}$

${ }^{\dagger}$ Department of Material Science and NanoEngineering, Rice University, Houston, Texas 77005, United States,

College of Chemistry and Chemical Engineering, Chongqing University, Chongqing 400044, P. R. China,

${ }^{\#}$ Applied Physics Department, State University of Campinas, Campinas-SP, 13083-959, Brazil.

${ }^{\dagger \dagger}$ Hysitron, Inc., Minneapolis, MN 55344

KEYWORDS: Carbon, Nanosphere, In-situ mechanical tests, Doping, Elastic Modulus

\begin{abstract}
Understanding properties of individual nanostructures, such as mechanical properties and deformation mechanism, aid to control their properties for specific applications. Here we report, the mechanical properties of individual boron and nitrogen doped carbon-based nanospheres (CNS) using in-situ nano-compression testing in a scanning electron microscopy (SEM). The insitu SEM characterizations showed classical sphere deformation during initial loading and it can be deformed till 40-50 percent. Elastic modulus of spheres is $33.3 \mathrm{GPa}$ which has been determined using unloading curves. The mechanical properties of CNS structures are quite
\end{abstract}


outstanding when it is compared to some other conventional nanomaterials such as polymerbased spheres and nanotube structures.

\section{INTRODUCTION}

During last two decades the discovery of carbon nanomaterials such as fullerene, carbon nanotube and graphene opened a new era due to their exciting properties [1-3]. Carbon-based nanomaterials with highly curved graphitic structures, high electrical conductivity, thermal stability, high oxidation resistance, and superior mechanical properties are desirable for a broad range of applications such as environmental, biomedical and energy applications [4-7]. Among nanostructured carbon-based materials carbon nanospheres (CNS) have attracted great attentions since they have outstanding chemical stability, low density, as well as high compressive strength [8-12]. CNS structures have a broad range of applications such as supercapacitors, Li-ion batteries, biosensors, catalyst and drug-delivery [13-15].

Production of CNS structures broadly can be categorized into two types, high temperature synthesis such as chemical vapor deposition (CVD), arc-discharge and the low temperature pyrolysis and catalytic decomposition of organic compounds. For example, Chen and co-worker reported production of CNS using microwave assisted reaction followed by thermal treatment in ammonia atmosphere [16]. Another work about carbon-based spheres reported by W. Xiong et al. They reported synthesis of carbon-based spheres using hydrothermal method and demonstrated as supercapacitor electrodes [15]. In all types of productions, it is one of the most important requirements to control their properties for specific applications. Toward this aim, the properties of single nanomaterials must be understood well. Understanding the deformation mechanism of individual CNS will help us in engineering the 3D structure. Nanoindentation is the only viable and effective approach to measure their mechanical properties of small-sized 
nanomaterials [17-21]. Till now variety of work about in-situ mechanical properties of nano/micro spheres have been reported [22-24]. In most of these reports, AFM has been used to measure mechanical properties of the nanostructures. However the AFM tip and its geometry are critical in such test and need further processing using several models to incorporate its affect, which leads to an indirect measurement of the modulus value [13, 14]. For example, Armini and co-worker employed AFM tip to measure mechanical properties of polymethylmethacrylate (PMMA) polymers and polymer coated silica-shell particles [25]. Another work involving compression of polymer-based microspheres using AFM tip has been reported by Tan and coworkers [26]. They calculated the surface modulus of polystyrene particles from the deformation of microspheres using AFM tip [26]. In addition of AFM, in-situ SEM and TEM can be used to measure mechanical properties of individual nanomaterials [27-29]. For example, Shan et. al. used in-situ compression test in a TEM to determine mechanical properties of CdS nanoparticles [28]. They showed that high stress and strain of the CdS spheres are the result of their hierarchical structures. In the other report, Hao et. al. used in-situ a hybrid scanning electron microscope/scanning probe microscope (SEM/SPM) system to obtain the force-displacement curves and they calculated Young's modulus of microspheres based on Hertz's contact theory mechanics. Their results showed that Young's modulus of a polycrystalline $\mathrm{TiO}_{2}$ microspheres were approximately 30\% larger than the bulk materials [22]. Even there are a few reported studies, it still remains a big challenge to conduct mechanical properties of individual nanospheres. Here we report the mechanical testing of boron (B) and nitrogen (N) doped carbonbased single sphere using flat punch in-situ indenter attached with SEM.

\section{EXPERIMENTAL SECTION}

CNS structures in this report is produced using chemical vapor deposition (CVD) method. In typical CVD process, they are fabricated with introducing mixture of amonia borane, ferrocene 
in dichlorobenzene under $\mathrm{Ar} / \mathrm{H}_{2}$ as a carrier gases at $1000{ }^{\circ} \mathrm{C}$ (Figure 1a-c). As a result of the CVD reaction, boron (B) and nitrogen $(\mathrm{N})$ doped CNS structures produced and collected from the surface of the quartz tube. The obtained material was characterized using scanning electron microscopy (SEM), contact angle measurement, Raman spectroscopy, X-ray photoelectron spectroscopy (XPS), fourier-transformed infrared spectroscopy, thermogravimetric analysis (TGA), BET surface analyzer and transmission electron microscopy (TEM) and in-situ compression test in scanning electron microscope (SEM). Additionally, we demonstrate CNS structures as an anode material for Li-ion battery application.

\section{RESULTS AND DISCUSSION}

In ordered to understand the morphology of the obtained material is characterized with SEM. As shown in figure $1 \mathrm{~d}$ shows the entirely packed macroscopic structure of the acquired CNS. Figure 1e shows that the nano-sized interconnected CNS structures, thus these interconnection results highly porous framework which is superhydrophobic $\left(122^{\circ}\right)$ and light weight. Additionally, the macrostructure of CNS are perfectly shaped and consist of not only individual, also interconnected structures (Figure 1f). The diameters of spheres are ranging between $50 \mathrm{~nm}$ to $250 \mathrm{~nm}$.

The Raman spectra of CNS can be seen in figure 2a that shows two main peaks: the first peak is represents the graphitic layer (G-band) at $1592 \mathrm{~cm}^{-1}$, which is related to $\mathrm{sp}^{2}$ vibrations of CNS. The second peak at $1351 \mathrm{~cm}^{-1}$ (D-band) indicative of $\mathrm{sp}^{3}$ hybridization of CNS (Figure 1a) [30, 31]. The defect density on the surface of the structure can be determined from the ratio between $I_{D}: I_{G}$ bands. The relative intensity ratio of these two band $\left(I_{D}: I_{G}: 0.85\right)$ is regarded the degree of graphitization. XPS analysis was used for quantitative chemical analysis of CNS (Figure 2b). The C1s core level peak positions of the carbon atoms are at $283.7 \mathrm{eV}$. The peaks at binding 
energy near $284.3 \mathrm{eV}$ and $283.6 \mathrm{eV}$ are considered to be due to $\mathrm{B}-\mathrm{C}$ and $\mathrm{C}-\mathrm{N}$ bonding, respectively [32]. The Fourier transform infrared spectroscopy (FT-IR) of CNS shows in figure 2c. The peak at $912 \mathrm{~cm}^{-1}$ is attributed to B-N-B bending mode and the peak at $1382 \mathrm{~cm}^{-1}$ represents B-N stretching modes of BCN [33]. The peak at around $1071 \mathrm{~cm}^{-1}$ is corresponding to the B-C vibrations [34]. The peak around $1600 \mathrm{~cm}^{-1}$ split to two parts; the peak at $1604 \mathrm{~cm}^{-1}$ is corresponding to $\mathrm{sp}^{2} \mathrm{C}-\mathrm{N}$ bond vibration and the one at $1646 \mathrm{~cm}^{-1}$ is attributed to $-\mathrm{C}=\mathrm{C}-$ stretching $[35,36]$. Thermo-gravimetric analysis (TGA) data showing weight loss of CNS as a function of temperature between $30^{\circ} \mathrm{C}$ and $800^{\circ} \mathrm{C}\left(10{ }^{\circ} \mathrm{C} / \mathrm{min}\right.$, air atmosphere). The negative of the first derivative $\left(\%{ }^{\circ} \mathrm{C}\right)$ is also plotted as a function of temperature. As shown in figure $2 \mathrm{~d}$, the CNS is thermally stable until around $\sim 540{ }^{\circ} \mathrm{C}$ and $89 \%$ of the material decomposes until $\sim 660{ }^{\circ} \mathrm{C}$. The derivative weight loss as a function of the temperature shows that there are two overlapping steps to the CBN breakdown. These two steps show that the samples consist of two components which have similar decomposition temperature. Compared to some other carbon-based nanomaterials, CNS structures are thermally more stable. For example, decomposition temperature of some reported CNTs is around below $600{ }^{\circ} \mathrm{C}[37,38]$. BET surface analysis was conducted to determine the surface area, porosity of the CNS structures (Figure 2e). Porous materials can be classified in three categories; materials with pore diameter are less than $2 \mathrm{~nm}$ is microporous. If pore sizes are between 2 and $50 \mathrm{~nm}$, it is mesoporous and pore diameter larger than $50 \mathrm{~nm}$ is called macroporous. CNS samples were degassed for 18 hours at $200{ }^{\circ} \mathrm{C}$, and then BET surface analysis carried out. The $\mathrm{N}_{2}$ absorption can be classified as a type-I isotherm that exhibits the mesoporous structure of the CNS structures (Figure 2e). From the adsorption isotherm, the surface area of the CNS sample is $98.8 \mathrm{~m}^{2} / \mathrm{g}$. The inserted figure in figure $2 \mathrm{e}$ shows that the average pore diameter of CNS structure is $3.3 \mathrm{~nm}$ which point to mesoporous structures. 
In order to get detail information about CNS structures, detailed transmission electron microscopy (TEM) (Figure 3). As a result of TEM investigations, it clearly can be seen CNS are perfectly shaped (Figure 3a, b) and these nicely shaped spherical B and $\mathrm{N}$ doped carbon-based spheres are interconnected (Figure 3c). Besides TEM imaging, elemental mapping in TEM also performed to get information about distribution of doped elements. It clearly revealed the presence and uniform distribution of carbon, boron and nitrogen (Figure 3d).

Nanomechanical compression tests on individual CNS particles were conducted using an in situ SEM instrument, PI 85 PicoIndenter (Hysitron, USA) (Figure 4a). The particles were dispersed on Si substrate from solution, were compressed using $1 \mu \mathrm{m}$ diamond probe in load-control mode with a loading rate of $5 \mu \mathrm{N} / \mathrm{s}$ to a peak load of $100 \mu \mathrm{N}$. In all the tests, the load-displacement data and the real-time video of deformation were synchronized and captured simultaneously, which aided the post-experimental analysis. Figure 4b-c shows typical load-displacement data from $200 \mathrm{~nm}$ particles. The elastic modulus was determined from the unloading curves using [39].

$E=\left(1 / 2 r_{c}\right) \cdot d P_{d} / d h$,

Where $\mathrm{c}$ is the contact radius, $\mathrm{P}_{\mathrm{s}}$ is the applied load during unloading and $\mathrm{d}$ is the displacement during unloading. Using the equation, the average elastic modulus was calculated to be $33.3 \pm 1.5$ GPa. The sequence of images that are taken from the video is shown in figure 4c. The SEM images shows classical sphere deformation during initial loading and it can be deformed till 4050 percent. There is plastic deformation followed by fracturing can be observed at the top half the sphere. It can be observed that surface layer on the particles is peeled off from the core layer during deformation. The fractured shape clearly shows hollow structure inside. 
A comparative plot of the elastic modulus of CNS with polystyrene (PS) $(2.9 \pm 0.4 \mathrm{GPa})$ [8], $\mathrm{PS} / \mathrm{SiO}_{2}$ composite $(6.0 \pm 1.2 \quad \mathrm{GPa}) \quad[8], \quad \mathrm{PS} / \mathrm{CeO}_{2}$ composite $(7.93 \quad \mathrm{GPa})$ [40], polymethylmethacrylate (PMMA0 (4.3 $\pm 0.7 \mathrm{GPa})$ and $\mathrm{PMMA} / \mathrm{SiO}_{2}$ composite (4.5 \pm 0.7$)$ [25] shown in Figure 4d. . It clearly shows the elastic modulus of CNS is higher than above materials and very close to boron nitride nanotubes (BNNT) and carbon nanotubes (CNT) [41, 42].

The electrochemical measurements were performed by using 2032-type coin cells that were assembled in an argon-filled glove box with contents of oxygen and moisture below $0.1 \mathrm{ppm}$. The working electrodes were prepared by mixing the synthesized CNS and polyvinylidene fluoride (PVDF) as binder, and carbon black as a conductor with a weight ratio of 80:10:10 using n-Methyl-2-pyrrolidone (NMP) as the solvent. Then obtained slurry was coated onto a pure $\mathrm{Cu}$ foil $\left(99.6 \%\right.$, Good fellow) and dried in a vacuum oven at $85{ }^{\circ} \mathrm{C}$ for $12 \mathrm{~h}$. Lithium metal foil (Alfa Aesar) was used as a counter electrode. The liquid electrolyte used was $1 \mathrm{M} \mathrm{LiPF6}$ in a 1:1 v/v mixture of ethylene carbonate (EC) and diethyl carbonate (DEC) (Solvionic, France, battery grade). The charge/discharge performances were carried out on a LAND CT2001A battery system tester at various current rates in the voltage range of 0.01-3.0 V. The impedance spectra were recorded on an Autolab workstation (PGSTAT302N) by applying a sine wave with amplitude of $5.0 \mathrm{mV}$ over the frequency range from $100 \mathrm{kHz}$ to $0.01 \mathrm{~Hz}$.

The electrochemical properties of CNS as anode were evaluated using galvanostatic charge/discharge cycles with a potential window of $0.01-3 \mathrm{~V}$. Note that the specific capacity values reported here were calculated on the basis of the CNS weight. The charge/discharge profiles of the CNS samples in the $1^{\text {st }}, 2^{\text {nd }}$ and $100^{\text {th }}$ cycles at a current density of $50 \mathrm{~mA} \mathrm{~g}^{-1}$ are shown in Figure 5a. The initial discharge and charge capacity of the synthesized CNS are 413 and $320 \mathrm{mAh} \mathrm{g}^{-1}$, respectively. The initial irreversible capacity of $93 \mathrm{mAh} \mathrm{g}^{-1}$ results from the 
formation of solid electrolyte interface (SEI) film on the surface of carbon nanospheres as well as accompanied by electrolyte decomposition at a low potential region and the presence of active atoms such as nitrogen and boron [43]. After 20 cycles, the capacity is decreased to a minimum value of $300 \mathrm{mAh} \mathrm{g}^{-1}$ as shown in Figure 5b. However, it is interesting that a gradual increase of capacity is observed from that time on. After 100 cycles, a high capacity of $360 \mathrm{mAh} \mathrm{g}^{-1}$ is achieved. The extra capacity could arise from reversible reaction of lithium with active surface groups and dangling bonds. In addition, the long-term cycling performance and the corresponding Coulombic efficiency of the CNS at the currents of $50 \mathrm{~mA} \mathrm{~g}^{-1}$ and $250 \mathrm{~mA} \mathrm{~g}^{-1}$ are shown in Figure 5b. The prepared CNS as anode material demonstrate an excellent cycling stability around $270 \mathrm{mAh} \mathrm{g}^{-1}$ and a coulombic efficiency is close to $100 \%$ over 200 cycles. To understand the good electrochemical performance of the CNS anode, the electrochemical impedance spectroscopy (EIS) was conducted (Figure 5c). By fitting the Nyquist plot with Zview software by using a two-time constant equivalent. The inserted figure $5 \mathrm{c}$ represent the internal resistant of the test battery system. $\mathrm{R}_{\mathrm{f}}$ and $\mathrm{CPE}_{1}$ are associated with the resistance and constant phase element of the SEI film. $\mathrm{R}_{\mathrm{ct}}$ and $\mathrm{CPE}_{2}$ are associated with the charge-transfer resistant and constant phase element of the electrode/electrolyte interface. $Z_{\mathrm{w}}$ is associated with the Warburg impedance corresponding to the lithium-diffusion process. in lithium ion batteries, the charge transfer resistance is a measure of the charge transfer kinetics and the charge transfer process determines the rate of transfer reaction. It can be seen that the SEI film resistance $\left(\mathrm{R}_{\mathrm{f}}\right)$ and charge-transfer resistance $\left(\mathrm{R}_{\mathrm{ct}}\right)$ of the CNS electrode are 6.0 and $70.0 \Omega$, respectively. The small resistances further confirm the high conductivity and the excellent cycling stability of the CNS anode.

\section{CONCLUSION}


In conclusion, we have report scalable synthesis of boron and nitrogen doped carbon based nanospheres using CVD method. As a result of characterization, it has been shown that the mesoporous CNS consist of perfectly shaped nano-sized structures which are mostly interconnected. The in-situ compression test showed that the elastic modulus of CNS structure is 33.3 GPa which is much higher than reported PS spheres, PDMS spheres, PMMA spheres and their composites with metallic nanoparticles. Additionally, we have demonstrated as an anode material for Li-ion battery application.

Acknowledgment. This work has been supported by U.S. Department of Defense: U.S. Air Force Office of Scientific Research (AFOSR) for the project "MURI: Synthesis and Characterization of 3-D Carbon Nanotube Solid Networks" through the award \# FA9550-12-10035 and Air Force

Office of Scientific Research (AFOSR) for the project "Understanding Self-Strengthening Mechanisms in Nanocomposites” through Grant award \#FA9550-13-1-0084.

\section{AUTHOR INFORMATION}

\section{Corresponding Author}

E-mail: ajayan@rice.edu (P. M. Ajayan)

Email:sehmusozden@gmail.com (S. Ozden)

\section{References}

[1] H. Dai, Carbon Nanotubes: Synthesis, Integration, and Properties, Acc. Chem. Res. 35 (2002) 1035-1044. doi:10.1021/ar0101640 M4 - Citavi.

[2] L. Ma, A.H.C. Hart, S. Ozden, R. Vajtai, P.M. Ajayan, Advances of carbon nanomaterials, (2014) 9-46. doi:10.1039/C4FD90039A.

[3] A.K. Geim, K.S. Novoselov, The rise of graphene., Nat. Mater. (2007) 183-191. 
doi:http://dx.doi.org/10.1038/nmat1849.

[4] M. Mauter, M. Elimelech, Environmental applications of carbon-based nanomaterials, Environ. Sci. Technol. 42 (2008) 5843-5859. doi:10.1021/es8006904.

[5] C. Cha, S.R. Shin, N. Annabi, M.R. Dokmeci, Carbon-Based Nanomaterials : Multifunctional Materials for, ACS Nano. 7 (2013) 2891-2897. doi:10.1021/nn401196a.

[6] L. Dai, D.W. Chang, J.-B. Baek, W. Lu, Carbon nanomaterials for advanced energy conversion and storage., Small. 8 (2012) 1130-66. doi:10.1002/smll.201101594.

[7] Y.H. Ng, S. Ikeda, M. Matsumura, R. Amal, A perspective on fabricating carbon-based nanomaterials by photocatalysis and their applications, Energy Environ. Sci. 5 (2012) 9307. doi:10.1039/c2ee22128d.

[8] A. Chen, W. Mu, Y. Chen, Compressive elastic moduli and polishing performance of nonrigid core/shell structured PS/SiO2 composite abrasives evaluated by AFM, Appl. Surf. Sci. 290 (2014) 433-439. doi:10.1016/j.apsusc.2013.11.100.

[9] G. Zou, D. Yu, J. Lu, D. Wang, C. Jiang, Y. Qian, A self-generated template route to hollow carbon nanospheres in a short time, Solid State Commun. 131 (2004) 749-752. doi:10.1016/j.ssc.2004.07.004.

[10] A. Nieto-Marquez, R. Romero, A. Romero, J.L. Valverde, Carbon nanospheres: synthesis, physicochemical properties and applications, J. Mater. Chem. 21 (2011) 1664-1672. doi:10.1039/c0jm01350a.

[11] A.-H. Lu, T. Sun, W.-C. Li, Q. Sun, F. Han, D.-H. Liu, et al., Synthesis of Discrete and Dispersible Hollow Carbon Nanospheres with High Uniformity by Using Confined 
Nanospace Pyrolysis, Angew. Chemie Int. Ed. 50 (2011) 11765-11768.

doi:10.1002/anie.201105486.

[12] A.M. Herring, J.T. McKinnon, B.D. McCloskey, J. Filley, K.W. Gneshin, R. a Pavelka, et al., A novel method for the templated synthesis of homogeneous samples of hollow carbon nanospheres from cellulose chars., J. Am. Chem. Soc. 125 (2003) 9916-7. doi:10.1021/ja035031j.

[13] H. Cheng, B. Huang, Z. Wang, X. Qin, X. Zhang, Y. Dai, One-pot miniemulsionmediated route to $\mathrm{BiOBr}$ hollow microspheres with highly efficient photocatalytic activity, Chem. - A Eur. J. 17 (2011) 8039-43. doi:10.1002/chem.201100564.

[14] L.-H. Hung, S.-Y. Teh, J. Jester, A.P. Lee, PLGA micro/nanosphere synthesis by droplet microfluidic solvent evaporation and extraction approaches, Lab Chip. 10 (2010) 1820. doi:10.1039/c002866e.

[15] W. Xiong, M. Liu, L. Gan, Y. Lv, Y. Li, L. Yang, et al., A novel synthesis of mesoporous carbon microspheres for supercapacitor electrodes, J. Power Sources. 196 (2011) 1046110464. doi:10.1016/j.jpowsour.2011.07.083.

[16] T. Chen, L. Pan, T.A.J. Loh, D.H.C. Chua, Y. Yao, Q. Chen, et al., Porous nitrogen-doped carbon microspheres as anode materials for lithium ion batteries, Dalton Trans. 43 (2014) 14931-14935. doi:10.1039/C4DT01223B.

[17] W.C. Oliver, G.M. Pharr, An Improved Technique for Determining Hardness and ElasticModulus Using Load and Displacement Sensing Indentation Experiments, (1992).

[18] J. Chen, S.J. Bull, Relation between the ratio of elastic work to the total work of 
indentation and the ratio of hardness to Young's modulus for a perfect conical tip, J. Mater. Res. 24 (2009) 590-598. doi:10.1557/jmr.2009.0086.

[19] J. Chen, On the determination of coating toughness during nanoindentation, Surf. Coatings Technol. 206 (2012) 3064-3068. doi:10.1016/j.surfcoat.2011.12.006.

[20] J. Den Toonder, J. Malzbender, G. De With, R. Balkenende, Fracture Toughness and Adhesion Energy of Sol-gel Coatings on Glass, J. Mater. Res. 17 (2002) 224-233. doi:10.1557/JMR.2002.0032.

[21] Y.-T. Cheng, W. Ni, C.-M. Cheng, Determining the Instantaneous Modulus of Viscoelastic Solids Using Instrumented Indentation Measurements, J. Mater. Res. 20 (2005) 3061-3071. doi:10.1557/JMR.2005.0389.

[22] P. Hao, Y. Liu, Y. Du, Y. Zhang, Young 's Modulus of Polycrystalline Titania Microspheres Determined by In Situ Nanoindentation and Finite Element Modeling, 2014 (2014).

[23] E. Calvié, J. Réthoré, L. Joly-Pottuz, S. Meille, J. Chevalier, V. Garnier, et al., Mechanical behavior law of ceramic nanoparticles from transmission electron microscopy in situ nano-compression tests, Mater. Lett. 119 (2014) 107-110. doi:10.1016/j.matlet.2014.01.002.

[24] D.D. Stauffer, A. Beaber, A. Wagner, O. Ugurlu, J. Nowak, K. Andre Mkhoyan, et al., Strain-hardening in submicron silicon pillars and spheres, Acta Mater. 60 (2012) 24712478. doi:10.1016/j.actamat.2011.10.045.

[25] S. Armini, I.U. Vakarelski, C.M. Whelan, K. Maex, K. Higashitani, Nanoscale indentation 
of polymer and composite polymer-silica core-shell submicrometer particles by atomic force microscopy, Langmuir. 23 (2007) 2007-2014. doi:10.1021/la062271e.

[26] S. Tan, R.L. Sherman, W.T. Ford, Nanoscale compression of polymer microspheres by atomic force microscopy, Langmuir. 20 (2004) 7015-7020. doi:10.1021/la049597c.

[27] J. Deneen, W.M. Mook, A. Minor, W.W. Gerberich, C.B. Carter, In situ deformation of silicon nanospheres, J. Mater. Sci. 41 (2006) 4477-4483. doi:10.1007/s10853-006-0085-9.

[28] Z.W. Shan, G. Adesso, a Cabot, M.P. Sherburne, S. a S. Asif, O.L. Warren, et al., Ultrahigh stress and strain in hierarchically structured hollow nanoparticles., Nat. Mater. 7 (2008) 947-952. doi:10.1038/nmat2295.

[29] S.B. Hutchens, L.J. Hall, J.R. Greer, In situ mechanical testing reveals periodic buckle nucleation and propagation in carbon nanotube bundles, Adv. Funct. Mater. 20 (2010) 2338-2346. doi:10.1002/adfm.201000305.

[30] X. Sun, Y. Li, Colloidal Carbon Spheres and Their Core/Shell Structures with NobleMetal Nanoparticles, Angew. Chemie Int. Ed. 43 (2004) 597-601. doi:10.1002/anie.200352386.

[31] Y. Mi, W. Hu, Y. Dan, Y. Liu, Synthesis of carbon micro-spheres by a glucose hydrothermal method, Mater. Lett. 62 (2008) 1194-1196. doi:10.1016/j.matlet.2007.08.011.

[32] S.Z. Bai, B. Yao, G.Z. Xing, K. Zhang, W.-H. Su, Synthesis, conductivity and highpressure phase transition of amorphous boron carbon nitride, Phys. B Condens. Matter. 396 (2007) 214-219. doi:10.1016/j.physb.2007.04.007. 
[33] T. Sugino, Y. Etou, T. Tai, H. Mori, Dielectric constant of boron carbon nitride films synthesized by plasma-assisted chemical-vapor deposition, Appl. Phys. Lett. 80 (2002) 649. doi:10.1063/1.1436522.

[34] K. Shirai, S. Emura, S.I. Gonda, Y. Kumashiro, Infrared study of amorphous B1-xCx films, J. Appl. Phys. 78 (1995) 3392-3400. doi:10.1063/1.359967.

[35] J. Lü, H. Li, P. Zhu, X. Lü, Y. Li, Composited BCN/carbon fibers prepared by hotfilament chemical vapor deposition, Appl. Surf. Sci. 257 (2011) 4963-4967. doi:10.1016/j.apsusc.2011.01.004.

[36] Y.Z. Jin, C. Gao, W.K. Hsu, Y. Zhu, A. Huczko, M. Bystrzejewski, et al., Large-scale synthesis and characterization of carbon spheres prepared by direct pyrolysis of hydrocarbons, Carbon N. Y. 43 (2005) 1944-1953. doi:10.1016/j.carbon.2005.03.002.

[37] S. Ozden, C.S. Tiwary, A.H.C. Hart, A.C. Chipara, R. Romero-Aburto, M.-T.F. Rodrigues, et al., Density Variant Carbon Nanotube Interconnected Solids., Adv. Mater. 11 (2015) 1842-1850. doi:10.1002/adma.201404995.

[38] S. Ozden, T.N. Narayanan, C.S. Tiwary, P. Dong, A.H.C. Hart, R. Vajtai, et al., 3D Macroporous Solids from Chemically Cross-linked Carbon Nanotubes, Small. 11 (2015) 688-693. doi:10.1002/smll.201402127.

[39] D. Guo, G. Xie, J. Luo, Mechanical properties of nanoparticles: basics and applications, J. Phys. D. Appl. Phys. 47 (2014) 013001. doi:10.1088/0022-3727/47/1/013001.

[40] Y.-F. Chen, C. Ching, T.-S. Wu, C.-R. Wu, W.-T. Hsieh, H.-Y. Tsai, Balanophora spicata and Lupeol Acetate Possess Antinociceptive and Anti-Inflammatory Activities In Vivo 
and In Vitro., Evid. Based. Complement. Alternat. Med. 2012 (2012) 371273. doi:10.1155/2012/371273.

[41] M. Zheng, X. Chen, I.-T. Bae, C. Ke, C. Park, M.W. Smith, et al., Radial mechanical properties of single-walled boron nitride nanotubes., Small. 8 (2012) 116-21. doi:10.1002/smll.201100946.

[42] Y.H. Yang, W.Z. Li, Radial elasticity of single-walled carbon nanotube measured by atomic force microscopy, Appl. Phys. Lett. 98 (2011) 48-51. doi:10.1063/1.3546170.

[43] F. Han, Y. Bai, R. Liu, B. Yao, Y. Qi, N. Lun, et al., Template-Free Synthesis of Interconnected Hollow Carbon Nanospheres for High-Performance Anode Material in Lithium-Ion Batteries, (2011) 798-801. doi:10.1002/aenm.201100340. 

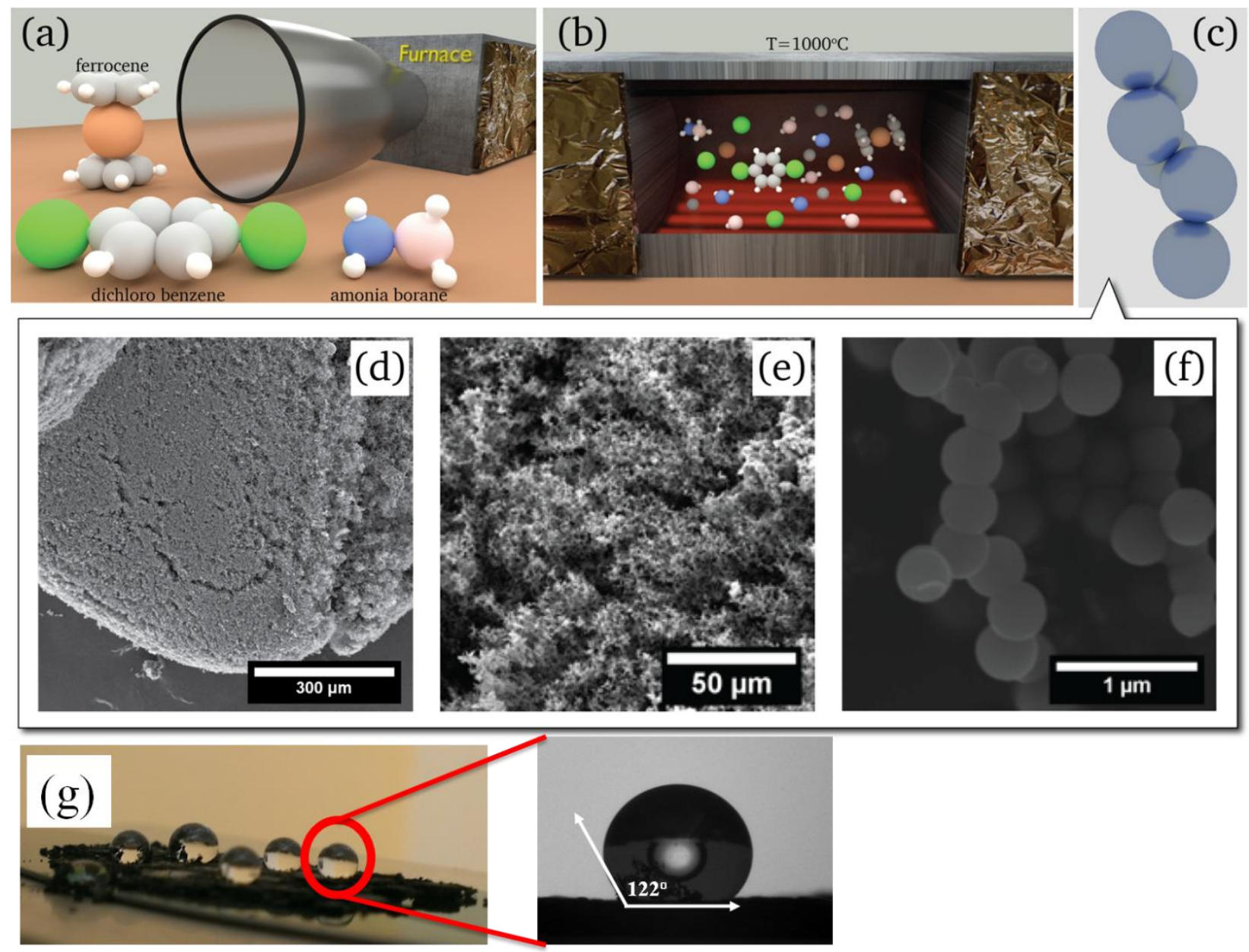

Figure 1. (a, b) Synthesis procedure of CNS, ammonia borane, ferrocene in dichlorobenzene under $\mathrm{Ar} / \mathrm{H}_{2}$ as a carrier gases purged to $\mathrm{CVD}$ at $1000^{\circ} \mathrm{C}$, (c) results $\mathrm{CNS}$, spheres . (d, e, f) scanning electron microscopy images of spherical structures. $(\mathrm{g})$ wettability properties of the as grown spheres. 

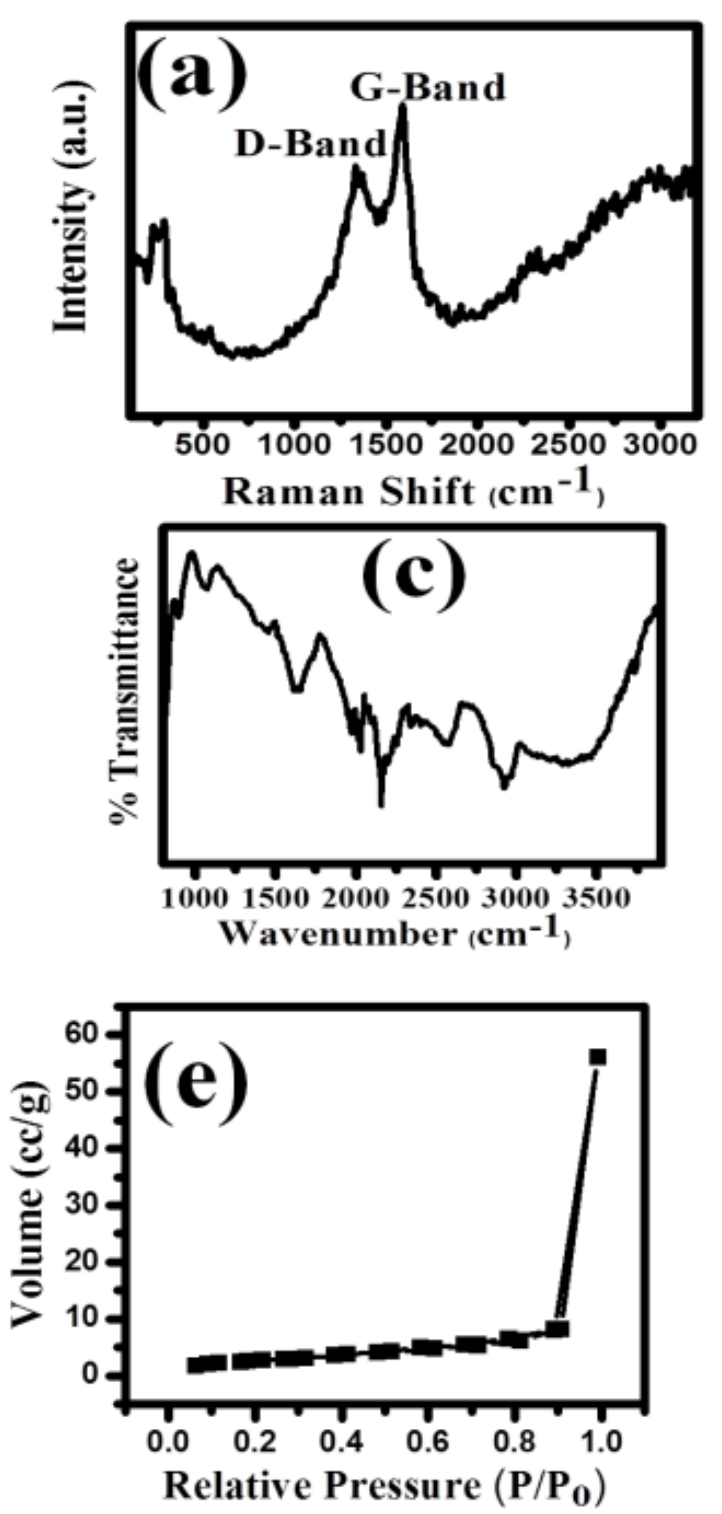
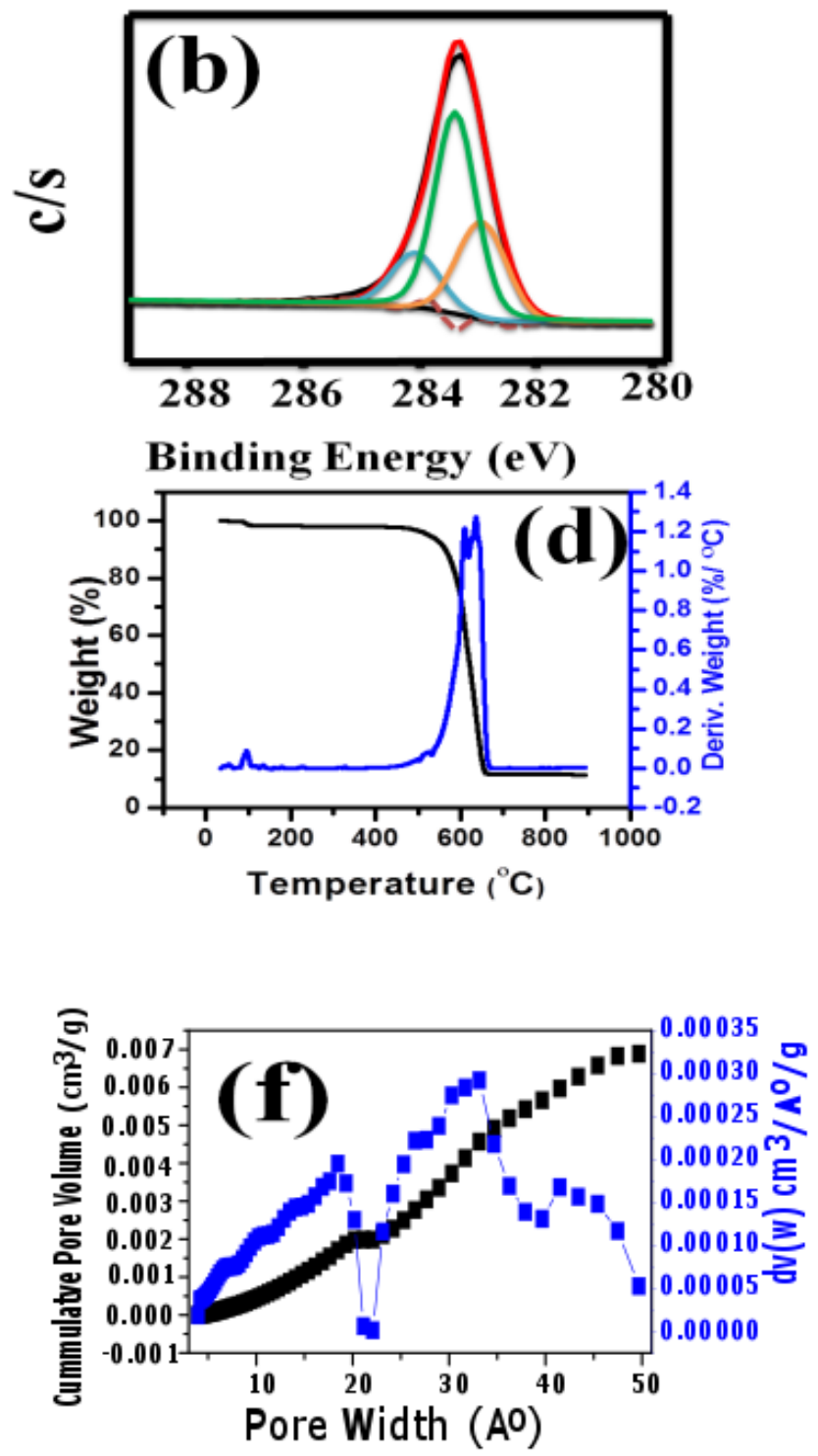

Figure 2. (a) Raman spectra, (b) XPS spectra, (c) FT-IR spectra of CNS, (d) thermogravimetric analysis of CNS, (e) The $\mathrm{N}_{2}$ absortion isotherm of spheres, (f) ) DFT method pore size distribution and cumulative pore volume of $\mathrm{CNS}$. 

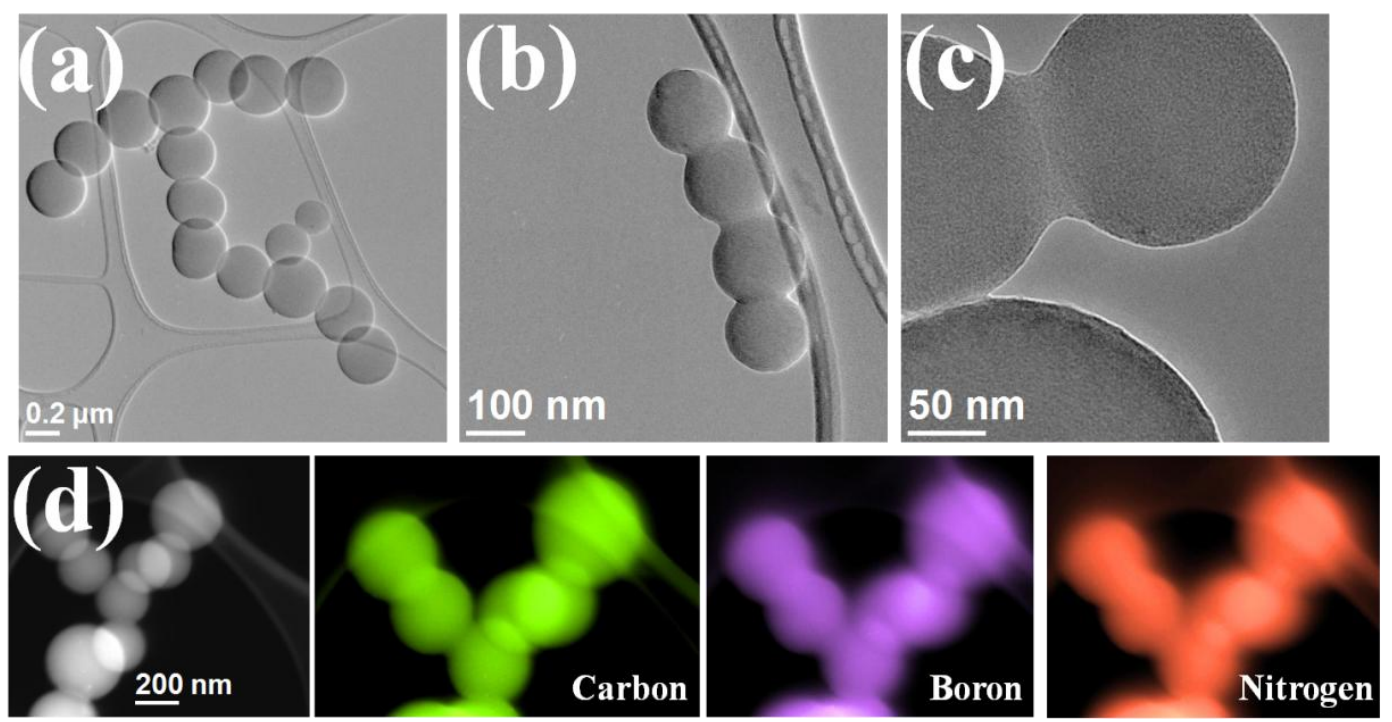

Figure 3. $(a, b, c)$ Transmission electron microscope images of $\mathrm{B}_{\mathrm{x}} \mathrm{C}_{\mathrm{y}} \mathrm{N}_{\mathrm{z}}$ spheres, it shows perfect spherical structures, (d) elemental mapping of TEM showing carbon boron and nitrogen distribution in the structure.
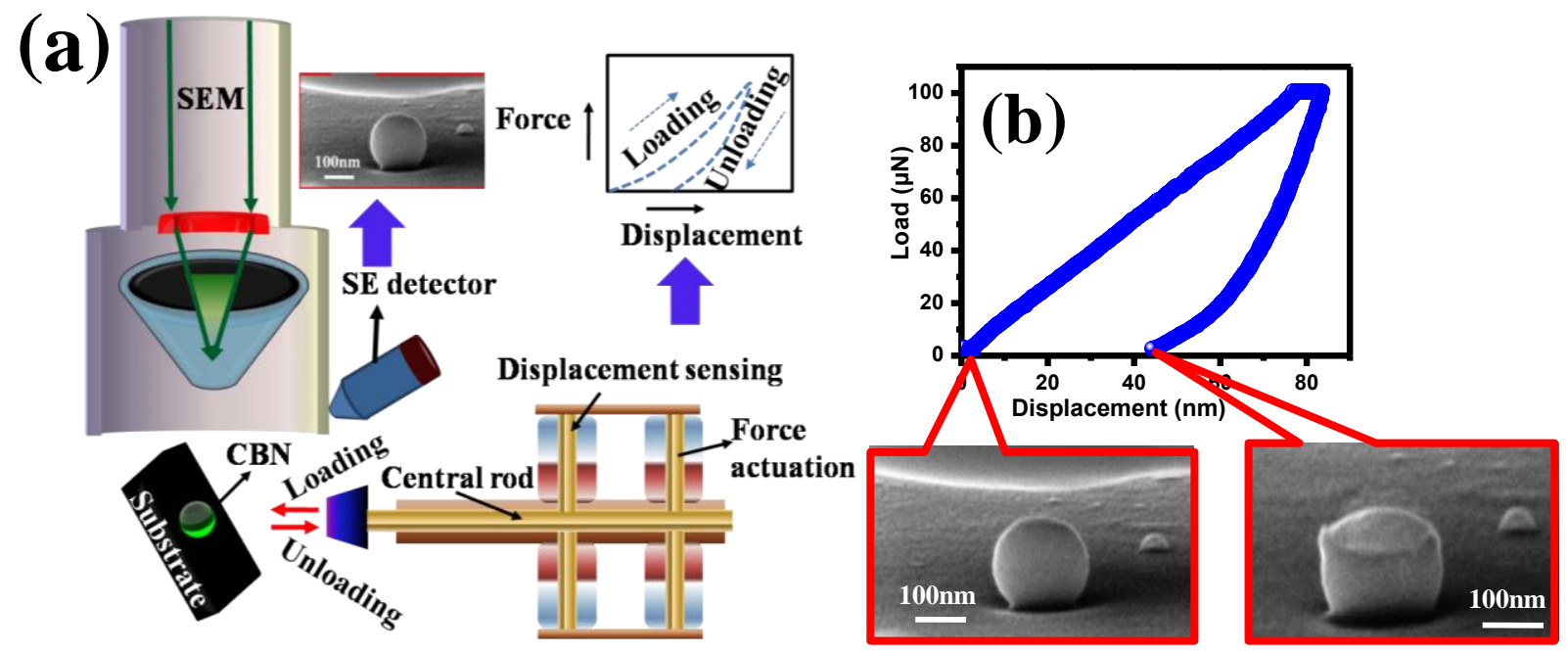

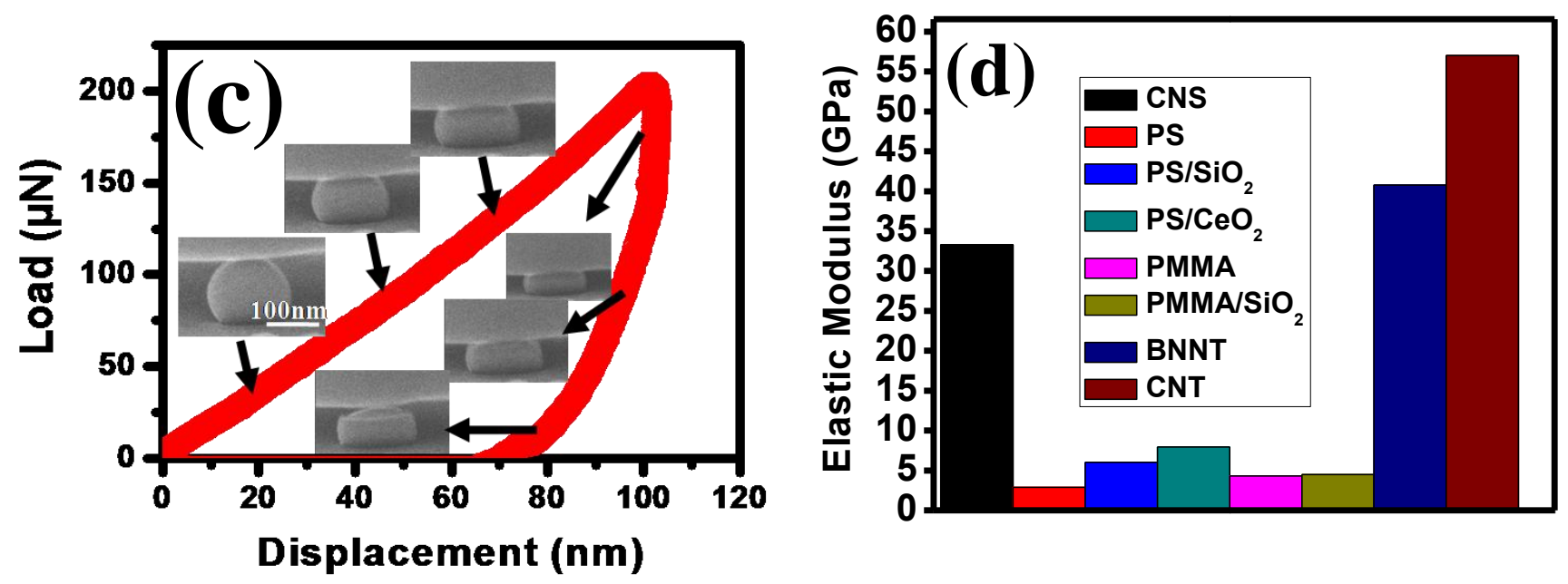

Figure 4. (a)Schematic showing in-situ SEM testing (b-c) Force-displacement curves extracted from nanoindentation experiments for different sized CNS structures, (d) comparison of elastic modulus of different types of nanomaterials with CNS structures. 

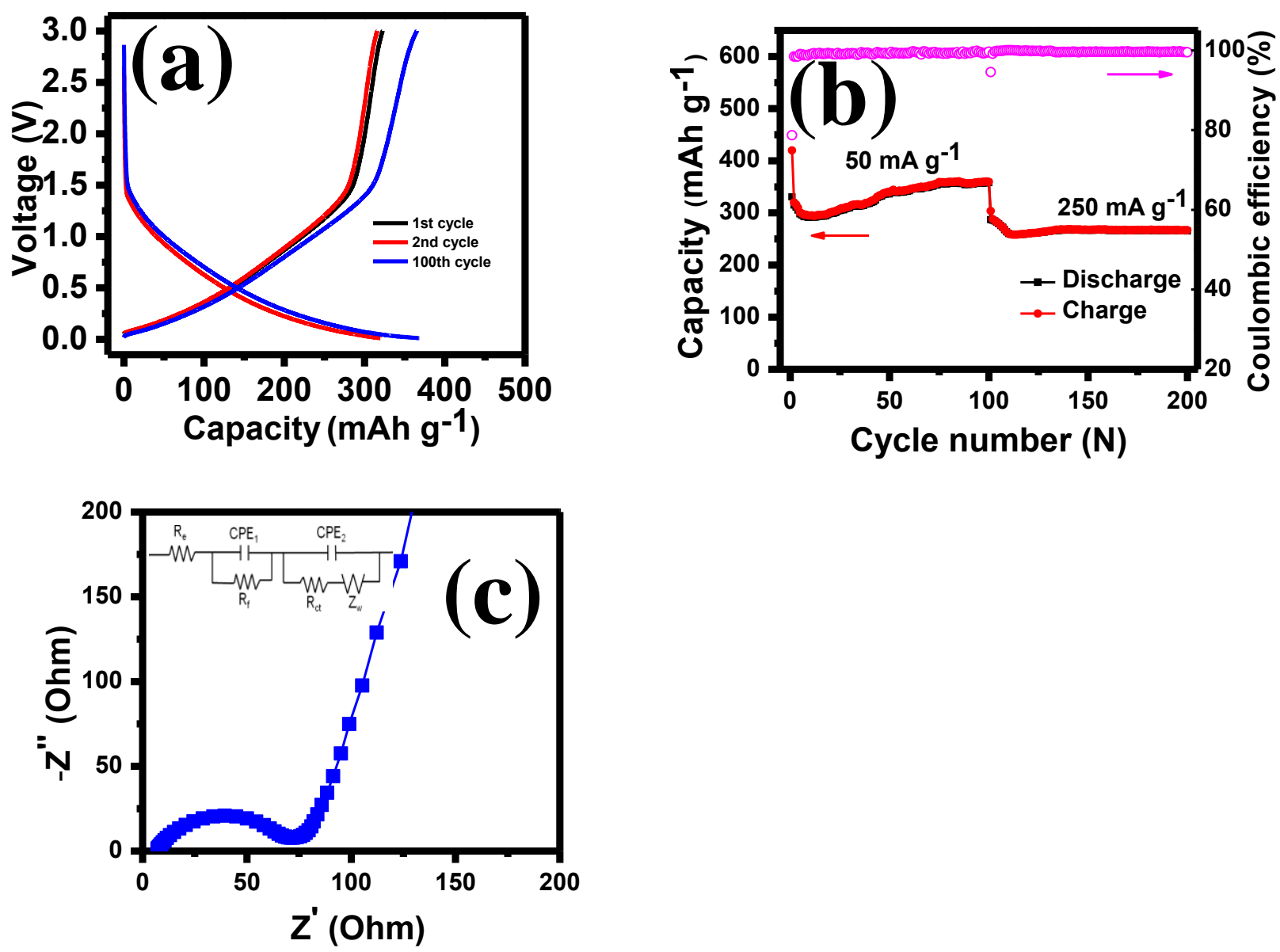

Figure 5. (a) Galvanostatic charge-discharge curves cycled at the 1 st, 2 nd, and 100th cycle of CNS between 3.0 and $0.01 \mathrm{~V}$ at a current density of $50 \mathrm{~mA} \mathrm{~g}-1$, (b) Cyclic performance of the CNS electrode cycled at current densities of $50 \mathrm{~mA} \mathrm{~g}-1$ and $100 \mathrm{~mA} \mathrm{~g} \mathrm{-1} \mathrm{(c)} \mathrm{Nyquist} \mathrm{plots} \mathrm{of}$ CNS obtained by applying a sine wave with amplitude of $5.0 \mathrm{mV}$ over the frequency range 100 $\mathrm{kHz}-0.01 \mathrm{~Hz}$. The inset in (c) is the equivalent circuit used in the simulation. 